\title{
IgA Monoclonal Gammopathy
}

National Cancer Institute

\section{Source}

National Cancer Institute. IgA Monoclonal Gammopathy. NCI Thesaurus. Code C128811.

An abnormal laboratory test result indicating the presence of IgA monoclonal

immunoglobulin in the blood or urine. 